\title{
一品生産の組立システムにおける生産データ分割に 基づく計画生成
}

\author{
荒井 栄司 ${ }^{* *} \cdot$ 内山 直樹 ${ }^{* * *}$
}

\author{
Manufacturing Data Separation Based Plan Generation \\ in Single Batch Assembly Systems*
}

Eiji Arai** and Naoki Uchiyama***

\begin{abstract}
This paper deals with a new manufacturing system for single batch production that will be a typical style in the next generation. Manufacturing data are divided into three types to reduce recalculation efforts against unpredicted events in the factory. They are product oriented data, facility oriented data and product and facility consideration data. The roles of process and operation planning, which have not been clearly defined yet, are distinguished according to the data separation. Due to this concept, a method to generate assembly plans is developed. First, representations of the plans and the manufacturing data for assembly are discussed. Then, a way to find all possible assembly plans with the divided manufacturing data is developed. A computational result shows the advantages of our method over the existing ones.
\end{abstract}

\section{Introduction}

In the conventional manufacturing systems, only host computer yields production plans and manufacturing facilities merely accomplish the previously determined works. Diversification of customer demands and increasing of computer abilities will lead the manufacturing style to the autonomous and distributed ones. Due to this tendency, we must look at some existing methods again and set forth a new production flows.

Several new architectures have been presented for the realization of autonomous manufacturing systems. Okino presented a Bionic Manufacturing System Modeler using a modeling element called as Modelon ${ }^{1}$. Ueda proposed a new concept for Bionic Manufacturing System based on DNA type information ${ }^{2)}$. Moriwaki et al. developed one of structures of components of autono-

* 原稿受付 1994 年 11 月 30 日

** 大阪大学 工学部 Department of Welding \& Production Engineering, Osaka University ; 2-1 Yamadaoka, Suita city, Osaka 565, JAPAN

*** 豊橋技術科学大学 工学部 Department of Mechanical Engineering, Toyohashi University of Technology ; 1-1 Hibarigaoka, Tenpakucho, Toyohashi city, Aichi 441, JAPAN

Key Words : FA, CAD/CAM, assembly planning, sub assembly, parts grouping. mous distributed manufacturing systems ${ }^{3)}$.

In this paper, we present one of the new manufacturing architectures for the single batch production that will be main style in the next generation. First, a manufacturing system based on the separation of manufacturing data is introduced, which can avoid the waste of recomputation time to cope with abrupt happenings in the factory. According to this concept, assembly planning system is designed as an example. New representations of the assembly plans, manufacturing data are presented. Then, a method of generating all possible assembly plans is shown. Finally, a computational example demonstrates the effectiveness of our method.

\section{Manufacturing data separation}

In the practical factory, there often occur unpredicted events such as failures of the machines, urgent orders. In such cases, though production plans generated already must be changed, most of the data need not to be modified, but part of them. Hence, dividing the data appropriately is significant to reduce the amount of computation to make the production plan to suit to the change in the factory. We classify manufacturing data into three types ; Product Oriented Data (POD), Facility Oriented Data (FOD) and 
Product and Facility Consideration Data (PFCD). The POD are defined as the data extracted only from the product, while the FOD refer the abilities of manufacturing facilities only, the PFCD consider both the product and the facilities' abilities. Fig.1 shows the outline of the proposed production planning flow based on the manufacturing data separation. The roles of process and operation planning systems, although they have not been clearly defined yet, are distributed according to the data separation. The process planning system extracts the POD. The operation planning system generates the PFCD referring to the POD and FOD. Each manufacturing facility has its own FOD. The plan generation system generates the final plans from the POD and PFCD. Obviously, this allotment of the data generation allows to reduce the computational efforts for some troubles of manufacturing facilities. Once the POD are extracted in the process planning system, it needs not to be used again for any breakdown of manufacturing facility because the POD are common to all facilities.

The data in each distributed data should also be stored separately as far as possible for the rapid plan modification.

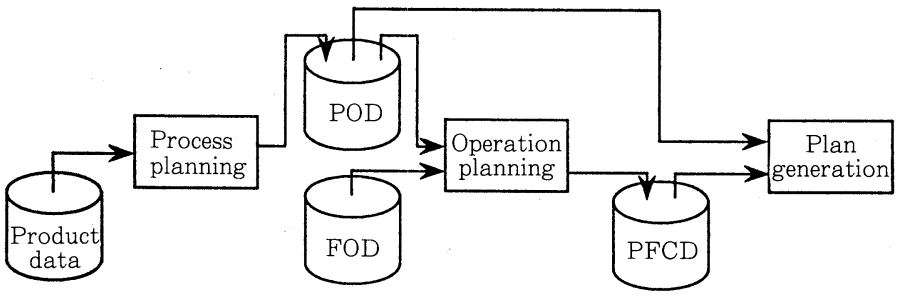

Fig. 1 Data separation based production planning

\section{Single batch assembly system}

We consider a single batch assembly system in which several AGVs and assembly cells work together as shown in Fig. 2. Each product to be assembled can be carried to any assembly cell by an AGV. Assembly plans for each product are

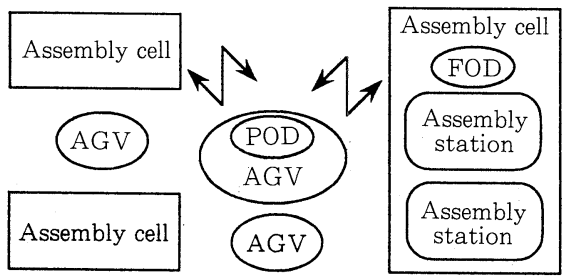

Fig. 2 Single batch assembly system generated by the communication between an AGV carrying that product and an assembly cell consisting of several assembly stations. Each AGV has POD for the carrying product. An AGV asks some of cells to assemble the product. Each cell generates the assembly plans according to the POD and its ability, then returns the plans to the AGV. The AGV selects the best assembly plan from the returned ones.

In this paper, the generation of assembly plans in each cell is focused on. From a survey of several studies concerning on assembly plan generation $^{4) \sim 6)}$, we have concluded that assembly planning systems must meet the following requirements :

(1) They can generate several plans for the alteration against the unpredicted events.

(2) They can reduce the number of generated plans not to exceed the computational limitations.

An antinomy between these two requirements forces the system to generate an appropriate number of plans. Considering these requirements, we propose a new assembly plan generation method for an assembly cell. That is under the following assumptions.

(1) Any two parts, two subassemblies, or part and subassembly that have been assembled can not be disconnected again during assembly operation.

(2) Each station is used just only one time.

In this study, a term 'subassembly' is used as a set of parts assembled from some smaller sets of parts by a station.

Fig. 3 shows an outline of the proposed method. First, it calculates the index of number of possible plans. If it is larger than predefined upper limit, the system reduce the number of plans by referring to other PFCD. By repeating this procedure, an appropriate number of plans can be obtained.

In this paper, the generation of assembly plans in each cell is focused on. Namely, we assume that the POD and the PFCD are both previously given. In the following section, firstly the representation of assembly plans, POD and PFCD are discussed. Then a method of generating all assembly plans that satisfy the given POD and PFCD is presented. 


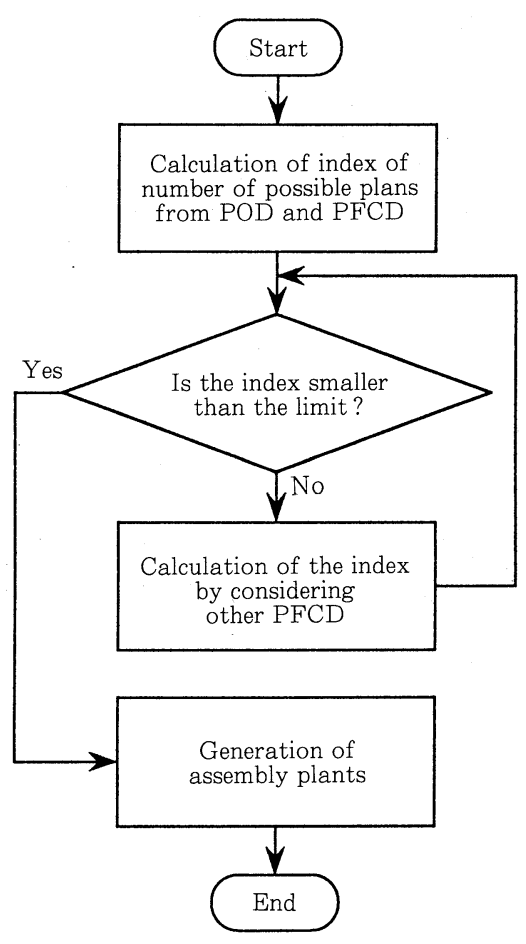

Fig. 3 Assembly plan generation flow

\section{Data separation based assembly planning}

\subsection{Representation of assembly plans}

We represent an assembly plan with a graph such as shown in Fig. 4 where $\boldsymbol{P}=\left\{p_{1}, \cdots, p_{n}\right\}$ and $\boldsymbol{E}=\left\{e_{1}, \cdots, e_{m}\right\}$ denote a set of parts constructing the product, a set of stations in an assembly cell respectively. This figure shows the case where $n=5, m=3$. The representation of the graph will be discussed in detail.

(1) A node of the graph denotes a part of the assembled products, named as a part node, or an assembly station in an assembly

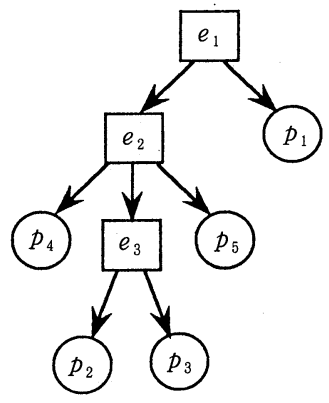

Fig. 4 Representation of assembly plan cell, named as a station node. An arc means that a station assembles a complete assembly or subassembly from several subassemblies and/or parts. The arc has an station node as an initial node, and a part node or a station node as a terminal node.

(2) Each part node corresponds to a leaf node of the graph, so that the out-degree of a part node is 0 since no part can perform the assembly operation. The in-degree of a part node is 1 because no part can be decomposed.

The graph satisfying above two conditions can be represented by the following matrix and constraints.

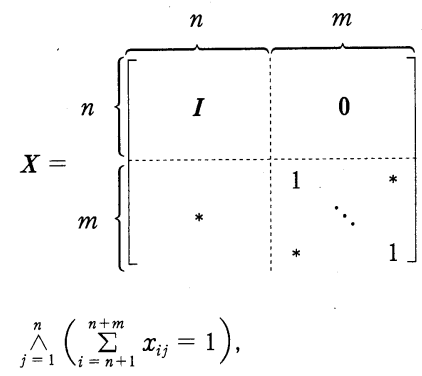

where $x_{i j}$ denotes an element of $\boldsymbol{X}$ and $\wedge$ is logical-and connection. All diagonal elements of the matrix in eq. (1) are 1 for the convenience. The elements of the upper left $n \times n$ submatrix denote the relations between parts, which are all 0 except diagonal elements because, in the graph, there is no arc whose initial and terminal nodes are both part nodes. The elements of the lower right $m \times m$ submatrix stand for the relation between stations, in which $x_{(n+r)(n+s)},(r \neq s)$ is 1 if an arc exist whose initial and terminal nodes mean station $r$ and station $s$ respectively. Both upper right $n \times m$ and lower left $m \times n$ submatrices represent the relations between parts and stations. In the former, all elements are 0 because there is no arc whose initial node denotes a part and terminal nodedenotes a station. In the latter, $x_{(n+r) s},(r \neq s)$ is 1 if an arc exist whose initial and terminal nodes correspond to station $r$ and part $s$ respectively.

(3) There is only one node coinciding with the root node in the graph. The in-degree of the root node is 0 , while those of all other nodes are 1.

There is one station generating the complete assembly at last. The station corresponds to a root node whose in-degree is 1 . On the other hand, from the assumption that no part nor subassembly, which has been assembled at once, can be 
decomposed again throughout the assembly operation, the in-degrees of all nodes are 1 . The condition (3) can be represented as follows :

$$
\begin{aligned}
& \bigwedge_{j=n+1}^{n+m}\left(\sum_{i=n+1(\neq j)}^{n+m} x_{i j} \leq 1\right), \\
& \sum_{i=n+1}^{n+m} \sum_{j=n+1(\neq i)}^{n+m} x_{i j}=m-1 .
\end{aligned}
$$

(4) Out- degrees of all station nodes are more than 2 .

Assembling means that two or more different parts are connected into one, so that the out-degrees of all station nodes are 2 or over. This condition can be given by :

$$
\bigwedge_{i=n+1}^{n+m}\left(\sum_{j=1}^{n+m} x_{i j} \geq 2\right) \text {. }
$$

(5) The graph is a connected graph.

The graph must be connected so as to make the product to be assembled entirely. To represent this condition mathematically, let us first consider undirected graph. In the undirected graph, the relation among the number of cycles, arcs and nodes, $N_{l}, N_{a}$ and $N_{v}$ respectively, states as follows :

$$
N_{l}=N_{a}-N_{v}+1 \text {. }
$$

From eq. (2) and (4), $N_{a}$ and $N_{v}$ in the graph are $n+m-1, n-m$ respectively so that $N_{l}$ comes to 0 if and only if the graph is a connected graph. From the fact that in-degrees of all nodes are less than or equal to 1 , the directed assembly graph never have a cycle if it is connected. Hence the fact stated above also be true for the directed graph (Fig. 5).

Since out-degrees of part nodes are all 0 , it is possible for only station nodes to be included in a cycle. Accordingly, the condition (5) can be given by :

$$
\boldsymbol{C}^{m}=\mathbf{0},
$$

where the element $c_{i j}$ of the matrix $\boldsymbol{C}$ is defined as follows :

$$
c_{i j}= \begin{cases}0, & \text { if } i=j, \\ x_{(n+i)(n+j)}, & \text { otherwise. }\end{cases}
$$

The number of arcs $N_{a}$ do not have to satisfy only $N_{a}=n+m-1$ but also $N_{a} \geq 2 m$ from eq. (5).

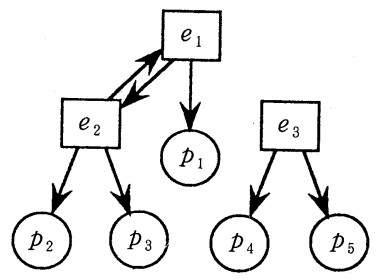

Fig. 5 Disconnected graph with a cycle
The following relation is derived :

$$
n+m-1 \geq 2 m \text {. }
$$

Therefore the number of parts $n$ is assumed as that more than $m+1$ throughout this paper for the sake of simplicity.

\subsection{Representation of POD and PFCD}

The POD are defined as the data extracted only from the product whereas the PFCD as those considering both the characteristics of the product and the manufacturing resource abilities. Let us first consider how to represent the PFCD. We use a set of parts constructing a subassembly to describe them. A condition, if any station can generate a certain subassembly, can be represented as a set of part nodes that are able to be reached from that station. For example, in Fig. 4, the station node $e_{2}$ can reach the part nodes $\left\{p_{2}, p_{3}, p_{4}, p_{5}\right\}$ by passing the arcs, then it can generate the subassembly $\left\{p_{2}, p_{3}, p_{4}, p_{5}\right\}$. Also the station node $e_{3}$ can reach the part nodes $\left\{p_{2}, p_{3}\right\}$, hence it can generate the subassembly $\left\{p_{2}, p_{3}\right\}$ from $p_{2}$ and $p_{3}$. Therefore the abilities of assembly facilities can be expressed by means of reachability matrix. Following two types of conditions are adopted as the PFCD.

(1) A station $e_{k}$ must generate a subassembly $s_{a}$ where $s_{a} \subseteq P$, the number of elements of $s_{a}$ is grater than or equal to $2, e_{k} \in E$. This condition can be represented as follows :

$$
\left(\bigwedge_{y=1}^{N\left(s_{a}\right)} x_{(n+k) q(y)}^{m}>0\right) \wedge\left({\stackrel{n-N\left(s_{a}\right)}{\bigwedge}}_{z=1}^{m} x_{(n+k) r(z)}=0\right)
$$

$x_{i j}^{m}$ is an element of $\boldsymbol{X}^{m}, N\left(s_{a}\right)$ denotes the number of parts included in $s_{a} . q(y)$ and $r(z)$ denote column numbers of the matrix corresponding to $y$ th element of $s_{a}, z$ th element of $P-s_{a}$ respectively.

(2) A station $e_{k}$ can not generate a subassembly $s_{a}$

This condition can be expressed as follows :

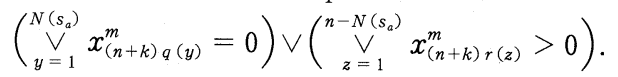

We prepare the following two conditions as the POD.

(3) A set of parts $s_{a}$ must be a subassembly

This condition can be represented by logicalor connections of eq. (7) for all stations.

(4) A set of parts $s_{a}$ can not be a subassembly

This condition can be represented by logicaland connections of eq. (8) for all stations.

For example, the following conditions can also be given by the combination of the above conditions : 
(5) A station $e_{k}$ must generate a subassembly from $s_{1}, \cdots, s_{h}$

This condition can be divided into following two :

$s_{1}, \cdots, s_{h}$ must be all subassemblies.

A station $e_{k}$ must generate a subassembly $\bigcup_{a=1}^{h} s_{a}$.

(6) A station $e_{k}$ must generate a subassembly from $s_{1}, \cdots, s_{h}$ and $p_{1}, \cdots, p_{g}$

This condition can be given by the combination of the following three :

Sets of parts $s_{1}, \cdots, s_{h}$ must be all subassemblies.

Any subset of $\left\{p_{1}, \cdots, p_{g}\right\}$ can not be a subassembly.

A station $e_{k}$ must generate a subassembly $\left(\bigcup_{a=1}^{h} s_{a}\right) \cup\left\{p_{1}, \cdots, p_{g}\right\}$.

\subsection{Generation of assembly plans}

By solving eqs. (2) (8) simultaneously, we can determine the values of all unknown elements in the matrix shown in eq. (1). It means that we can obtain the graphs representing the assembly plans. The solution can be computed with the method developed by Hammer and Rudeanu ${ }^{7)}$, however, if two or more stations without assigned task exist, several same graphs will be generated. This wastes the computational efforts. So we propose the method that can avoid generating the same graphs.

From eq. (2), only one element among the $n+1$ st through $n+m$ th rows is 1 for the 1 st through $n$th columns. From eqs. (3) and (4), all elements are 0 or only one element is 1 among $n+1$ st through $n+m$ th rows excepting diagonal elements for $n+1$ st through $n+m$ th columns. Based on this, a tree can be generated as shown in Fig. 6 which is for the case $n=4, m=3$. In

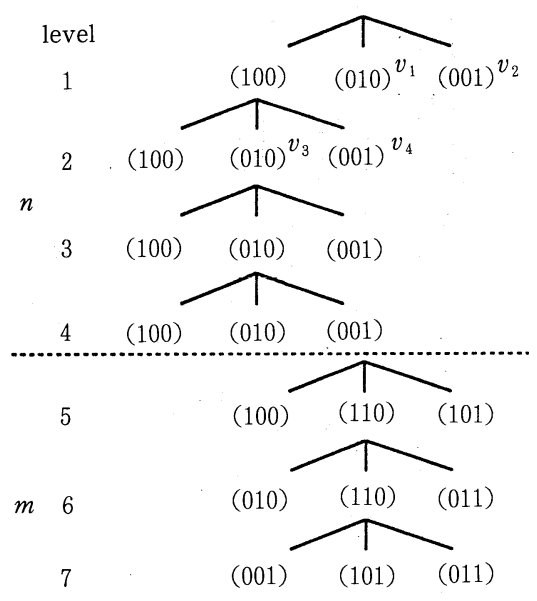

(a)

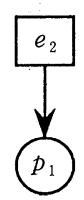

$v_{1}$

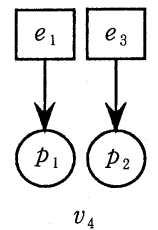

$v_{4}$

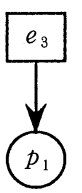

$v_{2}$

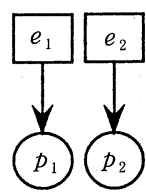

$v_{3}$

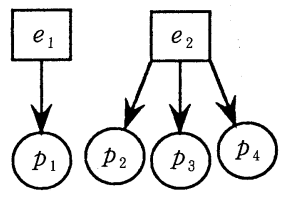

$v_{5}$ (b)

Fig. 6 Generation of assembly plans 
grater than or equal to 2 , the upper limit of the out-degree of one station node can be derived as follows :

$$
\sum_{j=1(\neq i)}^{n+m} x_{i j} \leq n+m-1-2(m-1)=n-m+1 .
$$

The number of nodes being generated can be reduced by eq. (9). In Fig. 6, for instance, the station node $e_{2}$ in $v_{5}$ is the initial node of three arcs, that does not satisfy eq. (9), so there is no need to generate the nodes inferior to $v_{5}$.

(3) Transformation of conditions about subassemblies

All nonlinear expressions involved in eqs. (6) (8) that are connected with logical-and or logical-or are used only to verify whether leftsides of them are more than 1 or equal to 0 . This fact is remarkable because we can transform them into either of following two :

$$
\begin{aligned}
& \sum_{i=1}^{u} a_{i} \prod_{j=1}^{v(i)} x_{i j}>0, \\
& \sum_{i=1}^{u} a_{i} \prod_{j=1}^{v(i)} x_{i j}=0 .
\end{aligned}
$$

where $u$ denotes the number of terms, $v(i)$ is the number of variables forming term $i, a_{i}$ is a positive integer. eq. (10) can not be satisfied when any one variable is 0 for all terms. Likewise, eq. (11) can not be satisfied if all variables in any one term are 1. Let us consider the following equation as an example:

$$
a_{1} x_{51} x_{52}+a_{2} x_{61} x_{62}+\cdots=0 \text {. }
$$

If all variables in the second term of eq. (12) come 1 during the generation of graphs, eq. (12) can not be satisfied. Therefore the searching space of the graphs can be decreased. Moreover, the more we give the condition about subassemblies, the more we reduce the computational efforts.

\subsection{Calculation of index on number of possible plans}

When insufficient number of conditions with respect to subassemblies are given, the number of graphs comes very large. This may require a great amount of conputation so that inappropriate graphs must be eliminated. For this, we introduce an estimation method of the number of feasible subassemblies which may allow the systems to decide wheter they should generate graphs or give more conditions about subassemblies. We shall explain this method with an example shown in Fig. 7.

Let $\boldsymbol{P}$ be a set of parts $\left\{p_{1}, \cdots, p_{9}\right\}$. Conditions that sets of parts, $s_{1}=\left\{p_{1}, \cdots, p_{6}\right\}, s_{2}=\left\{p_{1}, p_{2}, p_{3}\right\}$,

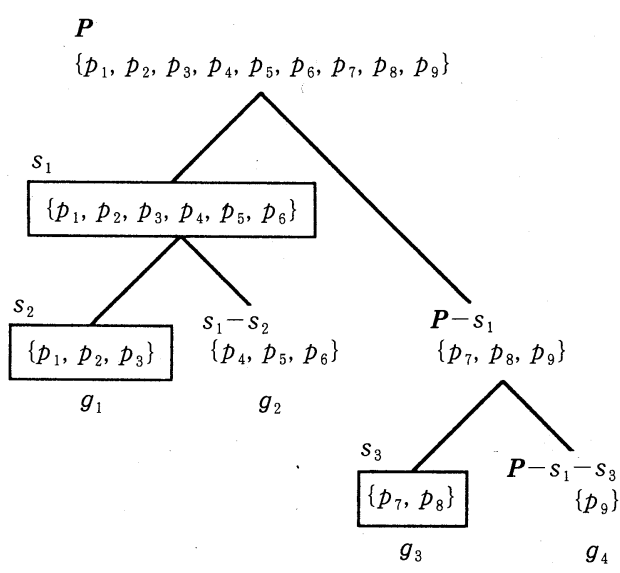

Fig. 7 Data generation to calculate the number of possible subassemblies

$s_{3}=\left\{p_{7}, p_{8}\right\}$ must be subassemblies respectively are given. First we execute $\boldsymbol{P}-s_{1}$ because $s_{1}$ has the largest number of parts of all subassemblies. Next we perform $s_{1}-s_{2}$ because $s_{2}$ is involved in $s_{1}$ and has the largest number of parts of the remains, and also perform $\boldsymbol{P}-s_{1}-s_{3}$ since $s_{3}$ is involved in $\boldsymbol{P}-s_{1}$ and has a largest number of parts of the remains. By repeating this procedure, a tree is created as shown in Fig. 7. In this figure, subassemblies surrounded by squares mean the given subassemblies. Let $g_{k},(k=1, \cdots, 4)$ be a set of parts corresponding to a leaf node in Fig. 7 .

By the definition of the graph representing assembly plan, any two subassemblies, $s_{a}$ and $s_{b}$, can exist together if and only if they satisfy following condition :

$$
\left(s_{a} \cap s_{b}=\phi\right) \vee\left(s_{a} \subset s_{b}\right) \vee\left(s_{b} \subset s_{a}\right) .
$$

With this condition and a graph as shown in Fig. 7, we can decide if arbitrary combination of $g_{k}$ can be a subassembly with a certain number of parts. For example, only subsets of $g_{1}$ or $g_{2}$ can be subassemblies consisting of two parts ( 2 parts subassembly) except $g_{3}$. Besides subassemblies made up of four parts (4 parts subassembly) can exist only as the combination of $g_{1}$ and elements of $g_{2}$. The results of applying this test for 2 through $n-1$ parts subassemblies are summarized in Table 1. The numbers of parts of subassemblies are listed in the left column. ${ }_{a} \mathrm{C}_{\mathrm{b}}^{k}$ denotes the number of choosing different combinations of $b$ parts from $a$ parts constructing $g_{k}$.

Total number of possible subassemblies can be calculated in the following manner. First we calculate the summation being over all elements listed in Table 1, then subtract the number of 
Table 1 Number of possible subassemblies

\begin{tabular}{|c|c|}
\hline $\begin{array}{l}\text { Number } \\
\text { of parts }\end{array}$ & Possible combination \\
\hline 2 & ${ }_{3} \mathrm{C}_{2}^{1} \quad{ }_{3} \mathrm{C}_{2}^{2} \quad{ }_{2} \mathrm{C}_{2}^{3}{ }^{s_{3}}$ \\
\hline 3 & ${ }_{3}{ }_{3} \mathrm{C}_{3}^{1} s_{2} \quad{ }_{3} \mathrm{C}_{3}^{2} \quad{ }_{2} \mathrm{C}_{2}^{3} \times{ }_{1} \mathrm{C}_{1}^{4}$ \\
\hline 4 & ${ }_{3} \mathrm{C}_{3}^{1} \times{ }_{3} \mathrm{C}_{1}^{2}$ \\
\hline 5 & ${ }_{3} \mathrm{C}_{3}^{1} \times{ }_{3} \mathrm{C}_{2}^{2}$ \\
\hline 6 & 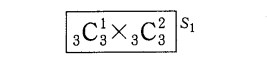 \\
\hline 7 & ${ }_{3} \mathrm{C}_{3}^{1} \times{ }_{3} \mathrm{C}_{3}^{2} \times{ }_{1} \mathrm{C}_{1}^{4}$ \\
\hline 8 & ${ }_{3} \mathrm{C}_{3}^{1} \times{ }_{3} \mathrm{C}_{3}^{2} \times{ }_{2} \mathrm{C}_{2}^{3}$ \\
\hline
\end{tabular}

given sets parts that must not be subassemblies and correspond to any of elements in Table 1 from the summation. For example, in the case that $\left\{p_{1}, p_{7}\right\}$ is given as a set of parts which must not be subassembly, the total number of possible subassemblies needs not to be reduced because $\left\{p_{1}, p_{7}\right\}$ does not correspond to any element.

\section{Example}

Let us consider the case that a product shown in Fig. 8 which have been used by De Fazio and Whitney ${ }^{4)}$ and an assembly cell consisting of four stations are both given. First we assume the following data are extracted in the process planning system as the POD :

$$
\text { (Condition 1) } \begin{aligned}
& \left\{p_{1}, p_{5}\right\},\left\{p_{1}, p_{6}\right\},\left\{p_{1}, p_{5}, p_{6}\right\}, \\
& \left\{p_{1}, p_{2}, p_{3}, p_{4}\right\},\left\{p_{2}, p_{3}, p_{4}, p_{6}\right\}, \\
& \left\{p_{1}, p_{2}, p_{3}, p_{4}, p_{6}\right\} \text { must not be } \\
& \text { subassemblies. }
\end{aligned}
$$

Because they are geometrically infeasible. The AGV carrying all parts of the product asks one cell to assemble them. The number of possible subassemblies is limited to 10 in the asked cell. The cell first estimates the number of possible

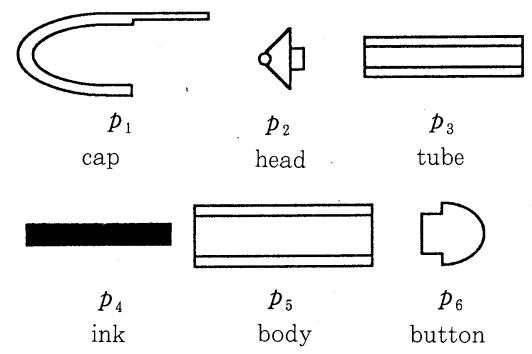

Fig. 8 Example of product subassemblies only from the POD. The number of possible subassemblies except the complete assembly is :

$$
N=50 \text {. }
$$

This number is grater than the limit. So the operation planning system in the cell generates the PFCD as follows :

(Condition 2) The station $e_{2}$ must generate the subassembly $\left\{p_{2}, p_{3}, p_{4}\right\}$ from $\left\{p_{2}, p_{3}\right\}$ and $p_{4}$.

The station $e_{2}$ is a special purpose machine which can inject ink into tube. A tree shown in Fig. 9 is generated for estimating the number of possible subassemblies. Table 2 shows all possible combinations of leaf nodes of the tree that can be subassemblies. The number of possible subassemblies except the complete assembly and given subassemblies becomes

$$
N=4 \text {. }
$$

This number is less than the limit so that the cell generates all assembly plans shown in Fig. 10. In this figure, white square means either of $e_{1}, e_{3}$ or $e_{4}$ which is not allocated specific tasks.

In this example, though if the cell will not able to be used from some reason, we will not need to regenerate Condition 1 . Though if any station except for $e_{2}$ will breakdown, Condition 2 will be still available. In such case, an assembly plan

\begin{tabular}{|c|c|}
\hline $\begin{array}{l}\text { Number } \\
\text { of parts }\end{array}$ & Possible combination \\
\hline 2 & $\begin{array}{ll}{ }_{2} \mathrm{C}_{2}^{1} & { }_{3} \mathrm{C}_{2}^{3} \\
\end{array}$ \\
\hline 3 & ${ }_{2} \mathrm{C}_{2}^{1} \times{ }_{1} \mathrm{C}_{1}^{2} \quad{ }_{3} \mathrm{C}_{3}^{3}$ \\
\hline 4 & ${ }_{2} \mathrm{C}_{2}^{1} \times{ }_{1} \mathrm{C}_{1}^{2} \times{ }_{3} \mathrm{C}_{1}^{3}$ \\
\hline 5 & ${ }_{2} \mathrm{C}_{2}^{1} \times{ }_{1} \mathrm{C}_{1}^{2} \times{ }_{3} \mathrm{C}_{2}^{3}$ \\
\hline
\end{tabular}

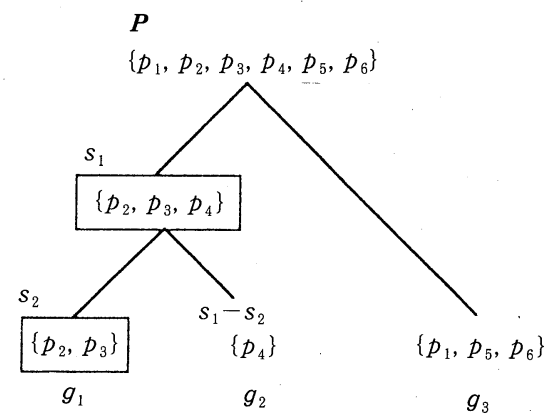

Fig. 9 Tree for calculating number of subassemblies of the example in Fig. 8

Table 2 Number of possible subassemblies of the example in Fig. 8 

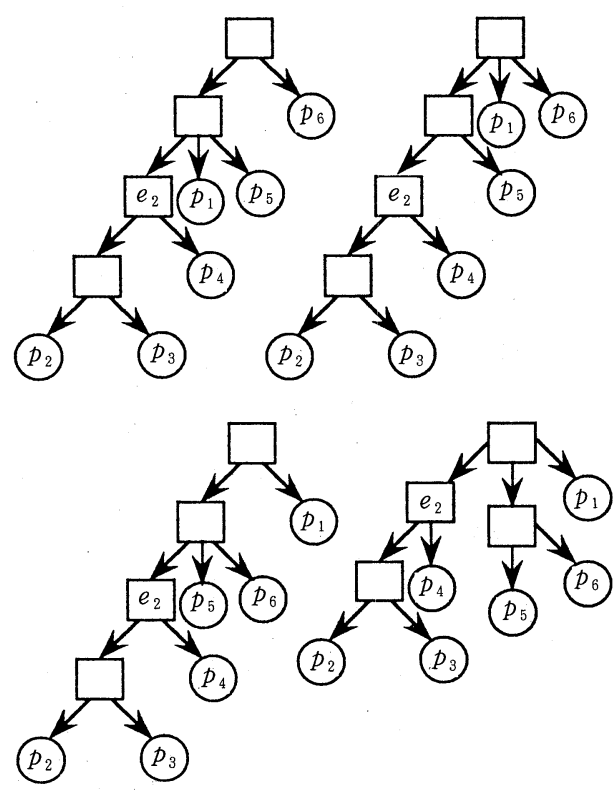

Fig. 10 Assembly plans for 4 stations

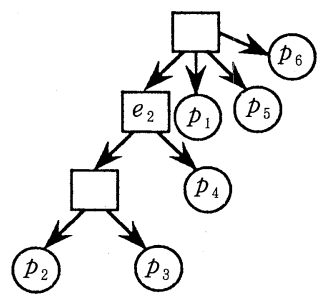

Fig. 11 Assembly plan for 3 stations

shown in Fig. 11 will be generated from the same POD and PFCD. Considering the computation time for the sudden happenings, manufacturing data should be stored separately as far as possible.

\section{Conclusions}

We presented a prototype assembly planning system on the basis of the manufacturing data separation into the POD, FOD and PFCD. There is no need to change all POD and part of PFCD against the failures of the facilities so that the data separation can reduce the amount of computation to cope with the unpredicted events in the factory.

Based on this concept, the methods to represent the assembly plans, POD and PFCD, and to generate the assembly plans by solving nonlinear Pseudo-Boolean equalities and inequalities are both developed. Our method can generate an appropriate number of assembly plans against the unpredicted changes and not to exceed the limitations of computational resources. Furthermore, we demonstrated the efficiency of the developed system through an example.

\section{References}

1) N. Okino: A Prototyping of Bionic Manufacturing System ; Proc. of the Int. Conf. on Object-Oriented Manufacturing Systems, pp. 297 302 (1992)

2) K. Ueda (G. J. Olling and F. Kimura (eds. )) : A Concept for Bionic Manufacturing Systems Based on DNAType Information, Human Aspects in Computer Integrated Manufacturing, Elsevier Science Publishers pp. 853 863 (1992)

3) T. Moriwaki, N. Sugimura, Y. Y. Martawirya and S. H. Wirjomartono: Autonomous Distributed Manufacturing System and Its Application to Scheduling Problem ; Proc. of the 1992 Pacific Conf. on Manufacturing, pp. 649 656 (1992)

4) T.L. De Fazio and D. E. Whitney : Simplified Generation of All Mechanical Assembly Sequences ; IEEE J. of Robotics and Automation, Vol. 3, No.6, pp. 640 658 (1987)

5) L. S. Homem de Mello and A. C. Sanderson : A Correct and Complete Algorithm for the Generation of Mechanical Assembly Sequences ; IEEE Trans. on Robotics and Automation, Vol. 7, No. 2, pp. 228 240 (1991)

6) C. L. Philip Chen : Automatic Assembly Sequence Generation by Pattern Matching ; IEEE Trans. Syst. Man and Cyberm., Vol. 21, No. 2, pp. 376 389 (1991)

7) P. L. Hammer and S. Rudeanu : The Theory of PseudoBoolean Programming, Lecture Notes in Mathematics, Springer, Vol. 23, pp. 57 83 (1966) 